\title{
Interleukin-17 in human inflammatory diseases
}

\author{
Arezoo Gowhari Shabgah¹, Ebrahim Fattahi², Fatemeh Zare Shahneh ${ }^{3,4}$ \\ ${ }^{1}$ Department of Immunology, School of Public Health, Tehran University of Medical Sciences, Tehran, Iran \\ Head of Department: Prof. Abbas Mirshafiey \\ 2Department of Immunology, Tabriz University of Medical Sciences, Tabriz, Iran \\ Head of Department: Dr. Jafar Majidi \\ ${ }^{3}$ Multiphase Chemistry Departments, Max Planck Institute for Chemistry, Mainz, Germany \\ Head of Department: Prof. Dr. Detlef Schuppan \\ ${ }^{4}$ Division of Translational Immunology, Department of Medicine I, University Medical Center, Johannes Gutenberg University, \\ Mainz, Germany \\ Head of Department: Prof. Dr. Ulrich Pöschl
}

\begin{abstract}
Human Th17 pro-inflammatory cells are currently defined as cells that produce IL-17A and F, tumor necrosis factor (TNF)- $\alpha$, IL-6, IL-21, IL-22 and IL-23. Recently discovered related molecules are forming a family of cytokines, the IL-17 family, IL-17A, IL-17B, IL-17C, IL-17D, IL-17E and IL-17F. The associated receptors for the IL-17 family identified are IL-17R, IL-17RH1, IL-17RL (receptor like), IL-17RD and IL-17RE. This review introduces the roles of IL-17 and Th17 cells in human autoimmune diseases. Studies have shown that T cells with inflammatory effects on epithelial, endothelial and fibroblast cells express IL-17. Th17 cells are supposed to be involved in various autoimmune diseases, such as rheumatoid arthritis, psoriasis, multiple sclerosis, and inflammatory bowel diseases. Base on the biologic functions and regulation, IL-17 has regulatory roles in host defense and chronic inflammation which result in tissue damage and autoimmunity. So the IL-17 links links innate and adaptive immunity and has both beneficial and pathological effects on the immune system. This paper will focus on the possible roles of IL-17 in autoimmune diseases, a fundamental player in immune regulation.
\end{abstract}

Key words: interleukin-17, T cells, inflammation, cytokines, cytokine receptors, autoimmunity.

\section{Identification of IL-17}

Two decades ago, Mossman and Coffman suggested that CD4+ T cells differentiate into two subsets with common functions and patterns of cytokine secretion, characterized by CD4+ Thelper 1 (Th1) or T helper 2 (Th2) cells. Th1 cells are designated by production of interleukin-2 (-2) and interferon- $\gamma$ (IFN- $\gamma$ ) and induction of cell-mediated immunity against intracellular pathogens and generation of delayed type hypersensitivity responses, while Th2 cells secrete interleukin-4 (IL-4), IL-5, and IL-13 through signal transducer and activator of transcription 4 (STAT4), and stimulate humoral immunity against parasitic helminthes [1].

Although CD4+ T-cell subpopulations such as T helper 3 (TH3) cells, T regulatory type 1 (TR1) cells, transforming growth factor (TGF)-induced regulatory T cells (iTregs), regulatory $T$ cells developed in the thymus (natural or nTregs) and follicular helper T (TFH) cells have been described, they are not defined as a separate lineage. IL-17 producing T cells represented a subset distinct from other CD4+ T-cell subsets, known as Th17. Th17 cells provide protection against some infection and are associated to the development of autoimmune diseases. Th17 cells as a pleiotropic cytokine can trigger an acute inflammatory response that is dominated by recruitment of granulocyte lineage, especially neutrophils against extracellular bacteria and some fungi [2]. IL-17 was defined as a proinflammatory cytokine produced by activated T-cells in response to stimulation through the T cell receptor. Th17 cells secrete not only IL-17A, but also IL-17F, IL-21, IL-22 and IL-23; these cytokines most likely cooperate to induce multiple inflammatory and hematopoietic effects on epithelial, endothelial, and fibroblastic cells [3].

Pathogens can induce Th17 response, including gram-positive Propionibacterium acnes, gram-negative Citrobacter rodentium, Porphyromonas gingivalis, Klebsiella pneumoniae, bacteroides species and Borrelia species,

Address for correspondence: Fatemeh Zare Shahneh, Multiphase Chemistry Departments, Max Planck Institute for Chemistry, Mainz, Germany Division of Translational Immunology, Department of Medicine I, University Medical Center, Johannes Gutenberg University, Mainz, Germany, phone (+49) 06131/305 7005, e-mail: f.shahneh@mpic.de

Received: 30.08.2013, accepted: 25.11.2013. 
Mycobacterium tuberculosis, parasitic infections like Toxoplasma gondii, and fungi such as Candida albicans [4, 5]. Now, several autoimmune diseases are supposed to be Th17-mediated diseases, because the biologic functions of IL-17 are consistent with the chronic and destructive nature of inflammation. This review introduces the roles of IL-17 and Th17 cells in human autoimmune diseases.

\section{The IL-17 family and its receptors}

IL-17 is a prototype member of the IL-17 family of cytokines, which contains six structurally related isoforms: IL-17A, IL-17B, IL-17C, IL-17D, IL-17E (IL-25), and IL-17F that share $20-50 \%$ homology (conserved cysteines) to IL-17. IL-17E (or IL-25) is not produced by Th17 cells, but it is produced by Th2 cells. IL-25 induces the expression of Th2-type cytokines and chemokines such as CCL5 (RANTES) and CCL11 (Eotaxin) [6]. The human IL-17 gene was mapped on human chromosome $6 \mathrm{p} 12$. The gene product is a protein of 150 amino acids with a molecular weight of $15 \mathrm{kDa}$ and is secreted as a disulfide linked homodimer of 30-35 kDa glycoproteins. IL-17 polypeptide comprises a 19-amino-acid signal sequence followed by a 136-amino-acid mature fragment. It comprises at least one $\mathrm{N}$-linked glycosylation site and biochemically, IL-17 A migrates as a dimer, apparently through six cysteine residues that form intermolecular bond interactions [7].

In contrast to IL-17A, IL-17B appears to be a non-disulfide-linked dimer. Also unlike IL-17A, IL-17B causes a dose-dependent recruitment of neutrophils to the peritoneal cavity following an injection of IL-17B into this site. IL-17C is expressed at very low levels; IL-17C induces secretion of IL-6 in human fibroblasts. IL-17D shares the most sequence homology with IL-17B (27\% sequence). Similar to IL-17A, the secretion of IL-6, IL-8, and GM-CSF in target cells can be triggered after IL-17D stimulation as a proinflammatory response. IL-17E (IL-25) has also a 25-35\% homology with other IL-17 family members [8]. Moreover various cell types, as well as $\gamma \delta$ T cells, NKT cells, NK cells, neutrophils, and eosinophil produce both IL-17A and IL-17F. Meanwhile IL-17B, IL-17C, IL-17D, and IL-17E cytokines are expressed from a wide range of tissues. Both IL-17A and IL-17F have proinflammatory properties and act on a broad range of cell types to induce the expression of cytokines (TGF- $\beta$, TNF, IL-1 $\beta$, IL-6, IL-21, IL-23, GMCSF and G-CSF), chemokines (CXCL1, CXCL8, CXCL10), and metalloproteinase. Furthermore, human Th17 cells produce CCL20 and CCR6 themselves [9].

IL-17A and IL-17F are also key cytokines for the recruitment, activation, and migration of neutrophils. IL-17, through modulation of chemokine activity, was described as a factor, which leads to the formation of germinal centers (GC) of lymph follicles containing B cells within GCs and increasing somatic hyper mutation. Follicular helper cells express CXCR5, respond to the lymph follicle, associated with chemokine CXCL13 and home, and help create the light zone of GCs, where further help can be given from cognate B cells that have undergone immunoglobulin isotype switching and somatic hyper mutation in the GC dark zone. Thus, T follicular helper cells in the $G C$ light zone induce further differentiation and selection of B cells. Both Th17 and T follicular helper cells are the main source of IL-21, and thus both play a significant role in setting up productive GC reactions [10, 11].

The human IL-17R gene has been mapped with clusters on chromosome 3. IL-17R complementary DNA (cDNA) encodes a single-pass transmembrane protein of approximately $130 \mathrm{kDa}(866 \mathrm{AA})$. The molecule consists of an N-terminal signal peptide (27-amino-acids) followed by a 293-amino-acid extracellular domain, a 21-amino-acid transmembrane domain, and an unusually long cytoplasmic tail of 525 amino acids. The IL-17R chain contains at least seven $\mathrm{N}$-linked glycosylation sites, and the molecular mass of nascent IL-17R protein is about $128-132 \mathrm{kDa}$. The IL-17 receptors family comprises IL-17RA, IL-17RB, IL-17RC, IL-17RD, and IL-17RE. IL-17RA was only responsible for signaling of IL-17A and IL-17F. However, recently IL-17RC has been identified to be necessary for the biological effects of IL-17A and IL-17F [12].

The receptor for IL-17A (type I trans-membrane glycoprotein receptor) seems to comprise two IL-17RA subunits and one IL-17RC subunit. IL-17RC exists in a large number of splice isoforms that differ in the extracellular domain. IL-17RA is expressed in hematopoietic tissue highly in response of IL-17A in epithelial, endothelial, fibroblast cells, macrophages, and DCs [13]. IL-17RB binds to IL-17B with a relatively high affinity. The IL-17RB protein has $19.2 \%$ protein sequence identity to the human IL-17RA sequences. IL-17RB signaling promotes Th2-type immunity. IL-17RC (IL-17RL) is 22\% identical and 34\% similar to the human IL-17RA with molecular weights of 33-60 kDa. IL-17RD reveals a remarkable relationship between the IL-17R family and conserved molecules involved in embryonic development. Whereas IL-17RA and IL-17RC are the receptors for IL-17A and IL-17F, IL-17RB is not only the receptor for IL-17E (IL-25) but also binds IL-17B with low affinity [14].

IL-17R family members have one transmembrane domain and a large intracellular C terminus. IL-17 seems to be activated by mitogen-activated protein kinase (MAPK) pathways and NF- $\mathrm{BB}$ via TRAF-6. IL-17RA binds both IL-17A and IL-17F, although it binds to IL-17A with higher affinity. IL-17RA is highly expressed on hematopoietic cells, but also minor on osteoblasts, fibroblasts, endothelial cells, and epithelial cells. In humans, IL-17RA can form a heterodimer with IL-17RC that binds human IL-17A and IL-17F. In contrast to IL-17RA, IL-17RC is expressed only at low levels on hematopoietic cells, but it is highly expressed on non-hematopoietic cells [10]. 


\section{Differentiation of Th17 cells}

Th17 cells as a distinct subpopulation of CD4+ T helper cells are specialized by the unique identification of differentiation factors and transcription factors. Tumor necrosis factor- $\alpha$ (TNF- $\alpha$ ), IL-1 $\beta$, transforming growth factor- $\beta$ (TGF- $\beta$ ) and IL- 6 can enhance Th17 differentiation [15]. IL-17 does not inhibit Th1 or Th2 differentiation so Th1 and Th2 cells typically dominate over Th17 cells. One of the ways in which TGF- $\beta$ can promote Th17 differentiation is suppressing the production of the inhibitory cytokines IFN- $\gamma$ and IL-4. TGF- $\beta$ synergizes with IL- 6 to induce expression of the transcription factor ROR $\gamma$ t, a key regulator of Th17 differentiation $[16,17]$. In humans, retinoic acid receptor-related orphan receptor- $\gamma \mathrm{t}(\mathrm{ROR} \gamma \mathrm{t})$, IFN-regulatory factor 4 (IRF-4), aryl-hydrocarbon receptor (AHR), and the transcription factor signal transducer and activator of transcription 3 (STAT3) expression are induced by IL-1 $\beta$, IL-6, and IL-23 [18-20].

\section{IL-17 signaling pathways}

Studies have demonstrated that IL-17 is capable of activating nuclear factor $\kappa \mathrm{B}(\mathrm{NF}-\kappa \mathrm{B})$ transcription factors in many cell types, including fibroblasts, macrophages, chondrocytes, intestinal epithelial cells, and colonic and pancreatic myofibroblasts [21]. The NF- $\mathrm{KB}$ has been activated in response to IL-17D, IL-17E and IL-17F. IL-17 receptor (IL-17R) activates extracellular signal-regulated protein kinase (ERK1 and ERK2), stress-induced c-Jun N-terminal kinases (JNK-1 and JNK-2), and mitogen-activated protein kinases (p38 MAPKs) pathways. These signaling pathways result in up-regulation of IL-6, IL-1 and NF- $\mathrm{B}$ [22].

\section{The cellular source of IL-17}

IL-17 cytokines are now known to be secreted by other cell types apart from CD8+ T cells, $\gamma \delta T$ cells, natural killer T (NKT) cells, natural killer (NK) cells, monocytes, macrophages, dendritic cells (DC), microglia, neutrophils, eosinophil, astrocytes, and oligodendrocytes. Thus, cells of both the innate and the adaptive immune systems as well as non-immune cells [23] produce IL-17A and IL-17F.

\section{Cytokines expressed by Th17 cells}

Th17 lineage expresses IL-21 after activation and autocrine IL-21 plays a significant role in RORyt and IL-17 expression [24]. After IL-6 or IL-21 induces IL-23 receptor expression, IL-23 in combination with TGF- $\beta$ can induce ROR $\gamma t$ and IL-17 expression. IL-6 upregulates IL-21, then both IL-6 and IL-21 deregulate IL-23 receptor, and eventually IL-23 acts to deregulate the effectors function and pathogenicity in Th17 cells [25]. Human IL-21 deregulated IL-17F and IL-17A production and T-cell proliferation and to counteract suppression by Tregs. Other pro-inflammatory cytokines secreted from human Th17 cells include TNF- $\alpha$, IL-22, and IL-26 [26]. A subset of Th17 cells co-expresses with IFN- $\gamma$ and IL-10, however IL-10, an anti-inflammatory cytokine is generally supposed to include Th2 cells and various types of Tregs [27]. IL-10 produced by Th17 cells may serve as an important protective role in limiting inflammation and tissue damage which is normally caused by IL-17. Both IL-17A and IL-17F induce the production of antimicrobial peptides ( $\beta$ defensin-2, S100A7, S100A8, and S100A9), mucins (MUC5B and MUC5AC) [28], cytokines like IL-6, granulocyte colony-stimulating factor (G-CSF), and granulocyte-macrophage colony-stimulating factor (GM-CSF), chemokines (CXCL1, CXCL5, IL-8, CCL2, and CCL7), and matrix metalloproteinase (MMP1, MMP3, MMP9, MMP12, and MMP13) from fibroblasts, endothelial cells, and epithelial cells [29].

IL-17A induces intercellular cell adhesion molecule 1 (ICAM-1) for granulocyte recruitment and inflammation in keratinocytes, IL-1 and tumor necrosis factor (TNF) in macrophages, inducible nitric oxide synthase (iNOS) and cyclooxygenase-2 (COX-2) in chondrocytes, and COX-2-dependent prostaglandin E2-mediated receptor activator of nuclear factor $\kappa \mathrm{B}(\mathrm{NF}-\kappa \mathrm{B})$ ligand (RANKL) expression in osteoblasts. Furthermore, IL-17A promotes stem cell factor (SCF) and G-CSF-mediated granulopoiesis. IL-17A is also involved in neutrophil recruitment with CXCL8 (IL-8), CXCL1 (Gro- $\alpha$ ), CXCL2 (MIP2), and CXCL5 (LIX). CCL5 (RANTES) is involved in recruiting T cells, monocytes, basophils, and eosinophils [30].

\section{Trafficking of Th17 cells}

Pro-inflammatory cytokines, chemokines and chemokine receptors produced by Th17 cells may result in the inflammation and pathogenesis that is mediated by activated T cells trafficking into involved tissues. Human Th17 cells express the chemokine receptor CCR6 from healthy peripheral blood and inflamed tissue. In addition, the majority of the RORC expression was restricted to the CCR6+CCR4+ population, with a small amount in the CCR6+ CXCR3+ population [31]. CCR6 which mediates homing to skin and mucosal tissues, plays an important role in recruitment of pathogenic T cells in IL-17-associated inflammatory diseases including psoriasis, inflammatory bowel diseases (IBD), and rheumatoid arthritis (RA). The CCR6 ligand CCL20 is overexpressed in stromal cells by IL-17; Th17 cells draw further Th17 and Th1 cells to inflamed tissues [32].

\section{IL-17 and human inflammatory diseases}

IL-17A in humans are associated with pathology in numerous autoimmune and inflammatory conditions, such as rheumatoid arthritis (RA), multiple sclerosis (MS), psoriasis, Crohn's disease, systemic lupus erythematosus (SLE), asthma, Behçet's disease, and hyper lgE syndrome [33]. 


\section{Rheumatoid arthritis}

The RA is considered as a chronic inflammation of the synovitis of the joints. The major characteristic of inflamed arthritic joints is proliferating synovial fibroblasts, joint and cartilage progressive destruction, infiltrating CD4+ T cells and autoantibody-producing plasma cells (rheumatoid factor and anti-cyclic citrullinated peptide (CCP) antibodies), increased numbers of innate immune cells as well as DC, granulocytes and macrophages and ectopic germinal centers (GC) in joints [34]. High levels of IL-17 were found in the rheumatoid synovium of patients with RA, promote both inflammation, and bone degradation [35]. Th17 cells induce the production of pro-inflammatory cytokines such as TNF- $\alpha$, IL-1 $\beta$ and IL-6 from cartilage, synoviocytes, macrophages and bone cells. Th17 cells up-regulate RANK ligand in osteoblasts, stimulate the activity of matrix metalloproteinase (MMP) 1, 2, 3, 9 and 13, extracellular matrix degradation of the joint, and bone resorption. The IL-17 also stimulates the expression of multiple chemokines such as IL-8/CXCL8, CXCL1 (KC/GRO- $\alpha$ ), CXCL2 (MIP2 $\alpha /$ GRO- $\beta$ ), CCL20 (MIP$3 \alpha)$, CCL2 (MCP1) and CCL7 (MCP3). These chemokines can recruit neutrophils, macrophages and lymphocytes to the synovium, thereby enhancing inflammation with more severe joint damage $[36,37]$.

\section{Psoriasis}

Psoriasis is a chronic inflammatory skin disorder with dermal hyperplasia and skin infiltration by immune cells, which have migrated from blood. The main histological features of psoriatic skin are epidermal keratinocyte hyper-proliferation, dermal angiogenesis, and infiltration of dendritic cells (DCs), T lymphocytes, neutrophils, monocytes and macrophages [38]. Both Th1 and Th17 cytokines have been found in psoriatic skin, as well as IL-17A, IL-17F, IL-19, IL-20, IL-22, IL-23, IL-24, IL-26 and TNF- $\alpha$ in serum and lesion skin. IL-17 and IL-22 synergistically unregulated expression of skin antimicrobial peptides such as $\beta$-defensin-2 (BD-2), S100A7-9 (psoriasin), cathelicidin (LL37) and S100A8/9 (calprotectin) which may cause more resistant to skin infections than people without psoriasis [39].

\section{Systemic lupus erythematosus}

Systemic lupus erythematosus (SLE) is a chronic multi-organ systemic autoimmune disease described by hyper gammaglobulinaemia and autoantibody to nuclear antigens result in inflammation and damage to multiple organs especially kidneys. Increased plasma levels of IL-17 have been identified in the serum of SLE patients as a consequence of too much IL-17 synthesis by CD4+ T cells and CD3+CD4-CD8- double negative (DN) T cells [40]. IL-17 and IL-23 can be detected in the kidneys of patients with lupus nephritis, which may contribute to renal failure [41]. IL-17 promotes B cell survival both alone and synergistically with B cell-activating factor (BAFF), to protect B cells from apoptosis, thereby increasing the number of autoantibody producing cells. Increased BAFF expression is found in $22-25 \%$ of SLE serum samples. Hence, the impact of IL-17 on $B$ cells may explain its role in contributing to SLE pathogenesis. Th17 differentiation by IL-6, IL-21, and IL-23 induce transcription of the ROR $\gamma t$ and increase phosphorylation of STAT3 in SLE patients [42].

\section{Inflammatory bowel disease and Crohn's disease}

The roles of Th17 cells in inflammatory bowel disease (IBD) and Crohn's disease (CD) are more complicated than in other autoimmune diseases [43]. In both diseases, chronic relapsing inflammatory disorder in the mucosa leads to destruction of the lamina, with complications such as perforations, and internal or external fistulas. The presence of lymphocytic infiltrates in the inflamed colonic mucosa has shown the involvement of IL-17 in the pathogenesis of inflammatory bowel disease (IBD) [44]. Furthermore, elevated serum IL-17 concentration was found in patients with active IBD in comparison to healthy individuals. The key cytokines IL-6, IL-8, IL-12, IL-17, IL-23, and MCP-1 were found overexpressed in lesions of Crohn's disease [45].

\section{Multiple sclerosis}

Multiple sclerosis (MS) is a CD4+ T cell-mediated autoimmune disease involving the central nervous system. Migration and accumulation of inflammatory immune cells from blood occurred through the brain blood barrier. Chronic inflammation of the brain leads to the destruction of myelin sheaths leading to a decrease in influx transmission and function loss. The expression of IL-6, IL-1 $\beta$, IFN- $\gamma$ and, TNF- $\alpha$ in monocytes, microglial cells, and astrocytes was associated with active MS [46]. IL-17 overexpressed in brain biopsies of MS patients was found in the blood and cerebrospinal fluid (CSF) of MS patients. Studies on pathogenic T-cell response in MS indicated recruitment of myeloid cells like monocytes/macrophages and neutrophils to the site of the inflammation and brain lesions by development of human Th17 cells dependent on IL-1 $\beta$ and IL-6, IFN- $\gamma$ and, TNF- $\alpha$ [47]. Memory T cells producing IL-17 and IL-22 disrupt the tight junctions of the blood-brain barrier and enable Th17 cells to migrate into the central nervous system (CNS) and cause neuronal loss [48].

\section{Systemic sclerosis}

Systemic sclerosis (SSc) is a progressive sclerosis of the skin, lungs, gastrointestinal tract and by microvascular abnormalities of the skin and visceral organs dysfunction which leads to progressive fibrosis, defined as scleroderma [49]. A higher expression of IL-17A and IL-17F 
has been demonstrated in peripheral blood and fibrotic lesions of the skin and lungs of patients with scleroderma. Furthermore, IL-17A and IL-17F can enhance the secretion of the proinflammatory and pro-fibrotic cytokines such as IL-1 $\alpha$, IL-6, IL-23, IL-8, chemokines (CXCL12 and its receptor CXCR4, CCL20), and adhesion molecules (L-selectin and ICAM-1) from fibroblasts and endothelial cells [50-53].

So, IL-17 as a key event in SSc pathogenesis induces synthesis of several cytokines and proteins engaged in tissue remodeling, proliferation and differentiation of $B$ lymphocytes leading to inflammation, auto-antibody production, auto-aggression, angiogenesis, and disturbed turnover of extracellular matrix, microvascular damage, and fibrosis $[54,55]$.

\section{Behçet's disease}

Patients with active Behçet's disease (BD) had significantly higher CD4 (+) CD25 (+) T cells compared with healthy controls. Noticeable increase in Th17 cells were identified in the peripheral blood of patients with active BD. Th17 cells regulate inflammation via production of distinct cytokines such as the IL-17 family. Previous studies confirmed that Th17 cells are pathological in several human autoimmune and inflammatory diseases [56]. Th17 cells predominantly produce IL-17A-F, IL-21, IL-22 and TNF- $\alpha$. IL- 6 and TGF- $\beta$ induce the differentiation of Th17 cells from naive T cells. A high level of TBX 21 (Th1), RORC (Th17) and Foxp3 (Treg) were confirmed in neuro-BD [57]. The presence of the IL-21 and IL17-A producing $T$ cells was demonstrated in the cerebrospinal fluid, brain parenchyma inflammatory infiltrates, and intracerebral blood vessels of patients with active BD and central nervous system involvement. IL-21 represents a promising objective for novel therapy in $\operatorname{BD}[58,59]$.

\section{Hyper IgE syndrome}

The hyper IgE syndrome, a primary immunodeficiency, is associated with very high levels of IgE, skin and lung manifestations, bone disorders and infections. IgE is the prototypic immunoglobulin produced under the action of the Th2 cytokines [60]. Recently, mutations in the STAT3 gene, one of a family of transcription activators, have been identified, which nullify the ability to increase Th17 response. Indeed defects in Th17 cells have been observed in these hyper IgE patients. These findings result from the balance between the Th17 and Th2 pathways, where the Th17 pathway inhibits the Th2 pathway and IgE production. Patients have relapsing Candida albicans and Staphylococcus aureus infection in the skin and lungs [61].

\section{Conclusions}

IL-17 and Th17 subset is a new revival of the contribution of some $T$ cells to chronic inflammation and extra-cellular matrix destruction. A developing list of diseases has been associated with IL-17 but the final demonstration of its contribution to disease pathogenesis is still unknown. Tools are now getting ready to test these concepts in the clinic. Recent studies support the notion that deregulated production of IL-17 and IL-21, cytokines produced by Th17 cells, may participate in the pathogenesis of autoimmune diseases. Knowledge of the molecular networks responsible for the regulation of this T helper cell subset is accumulating at a rapid pace. This information will undoubtedly be critical for the development of innovative therapeutic strategies aimed at targeting autoimmune diseases.

\section{Conflict of interest}

There is no conflict of interest.

\section{References}

1. Basu R, Hatton RD, Weaver CT. The Th17 family: flexibility follows function. Immunol Rev 2013; 252: 89-103.

2. Miossec P, Kolls JK. Targeting IL-17 and Th17 cells in chronic inflammation. Nat Rev Drug Dis 2012; 11: 763-76.

3. Fujimura K, Oyamada A, Iwamoto Y, et al. CD4 T cell-intrinsic IL-2 signaling differentially affects Th1 and Th17 development. J Leukoc Biol 2013; 94: 271-9.

4. Kolls J. The role of Th17 cytokines in primary mucosal immunity. Cyt Gro Fact Rev 2010; 21: 443-8.

5. Matsuzaki G, Umemura M. Interleukin-17 as an effector molecule of innate and acquired immunity against infections. Microbiol Immunol 2007; 51: 1139-47.

6. Zhi Chen, O'Shea J. Th17 cells: a new fate for differentiating helper T cells. Immunol Res 2008; 41: 87-102.

7. Awasthi A, Kuchroo V. Th17 cells: from precursors to players in inflammation and infection. Interl Immunol 2009; 21: 489-98.

8. Zúńiga LA, Jain R, Haines C, Cua DJ. Th17 cell development: from the cradle to the grave. Immunol Rev 2013; 252: 78-88.

9. Maddur MS, Miossec P, Kaveri SV, Bayry J. Th17 cells: biology, pathogenesis of autoimmune and inflammatory diseases, and therapeutic strategies. Am J Pathol 2012; 181: 8-18.

10. Korn T, Bettelli E, Oukka M. IL-17 and Th17 cells. Annul Rev Immunol 2009; 27: 485-517.

11. Bettelli E, Korn T, Kuchroo V. Th17: the third member of the effector T cell Trilogy. Curr Opin Immunol 2007; 19: 652-7.

12. Gaffen S. Structure and signaling in the IL-17 receptor superfamily. Nat Rev Immunol 2009; 9: 1-24.

13. Tsai HC, Velichko S, Hung LY, Wu R. IL-17A and Th17 cells in lung inflammation: an update on the role of Th17 cell differentiation and IL-17R signaling in host defense against infection. Clin Dev Immunol 2013; 2013: 267971.

14. Fouser $L$. Th17 cytokines and their emerging roles in inflammation and autoimmunity. Immunol Rev 2008; 226: 87-102.

15. Wilson NJ. Development, cytokine profile and function of human interleukin 17-producing helper T cells. Nat Immunol 2007; 8: 950-7.

16. Acosta-Rodriguez EV, Napolitani G. Interleukins 1 beta and 6 but not transforming growth factor-beta is essential for the differentiation of interleukin 17-producing human T helper cells. Nat Immunol 2007; 8: 942-9. 
17. Ivaylo I, Ivanov A, Liang Zhoua. Transcriptional regulation of Th17 cell differentiation. Sem Immunol 2007; 19: 409-17.

18. O'Connor RA, Cambrook H, Huettner K, Anderton SM. T-bet is essential for Th1-mediated, but not Th17-mediated, CNS autoimmune disease. Eur J Immunol 2013; 43: 2818-23.

19. Yang XO. STAT3 regulates cytokine-mediated generation of inflammatory helper T cells. J Biol Chem 2007; 282: 9358-63.

20. Mc Geachy M, Daniel J. Th17 Cell differentiation: the long and winding road. Immunity 2008; 28: 445-53.

21. Korn T. Th17 cells: effector T cells with inflammatory properties. Sem Immunol 2007; 19: 362-71.

22. Chang S, Dong C. Signaling of interleukin-17 family cytokines in immunity and inflammation. Cell Sign 2011; 23: 1069-75.

23. Hisakata Y. Current perspectives on the role of IL-17 in autoimmune disease. J Inflam Res 2010; 3: 33-44.

24. Korn T. IL-21 initiates an alternative pathway to induce proinflammatory TH17 cells. Nature 2007; 448: 484-7.

25. Nurieva R. Essential autocrine regulation by IL-21 in the generation of inflammatory T cells. Nature 2007; 448: 480-3.

26. Zheng Y. Interleukin-22, a Th17 cytokine, mediates IL-23-induced dermal inflammation and acanthosis. Nature 2007; 445: 648-51.

27. Rutz S, Eidenschenk C, Ouyang W. IL-22, not simply a Th17 cytokine. Immunol Rev 2013; 252: 116-32.

28. Becher B, Segal BM. Th17 cytokines in autoimmune neuro-inflammation. Curr Opin Immunol 2011; 23: 707-12.

29. Oh H, Ghosh S. NF-kappaB: roles and regulation in different CD4 (+) T-cell subsets. Immunol Rev 2013; 252: 41-51.

30. De Jong E, Suddason T, Lord G. M. Translational mini-review series on Th17 cells: induction of interleukin-17 production by regulatory T cells. Clin Exp Immunol 2009; 159: 109-19.

31. Louten J, Boniface K, Waal Malefyt R. Development and function of TH17 cells in health and disease. J Allergy Clin Immunol 2009; 123: 1004-11.

32. Muranski P, Restifo NP. Essentials of Th17 cell commitment and plasticity. Blood 2013; 121: 2402-14.

33. Tesmer M. Th17 cells in human disease. Immunol Rev 2008; 223: 87-113.

34. Li N, Wang JC, Liang TH, et al. Pathologic finding of increased expression of interleukin-17 in the synovial tissue of rheumatoid arthritis patients. Int J Clin Exp Pathol 2013; 6: 1375-9.

35. Iwakura Y. The roles of IL-17A in inflammatory immune responses and host defense against pathogens. Immunol Rev 2008; 226: 57-79.

36. Azizi G, Jadidi-Niaragh F, Mirshafiey A. Th17 cells in immunopathogenesis and treatment of rheumatoid arthritis. Int J Rheum Dis 2013; 16: 243-53.

37. Truchetet ME, Mossalayi MD, Boniface K. IL -17 in the rheumatologist's line of sight. Biomed Res Int 2013; 2013: 295132. doi: 10.1155/2013/295132

38. Raychaudhuri SP. Role of IL-17 in psoriasis and psoriatic arthritis. Clin Rev Allergy Immunol 2013; 44: 183-93.

39. Arican O, Sasmaz S, Ciragil P. Serum levels of TNF-alpha, IFN-gamma, IL-6, IL-8, IL-12, IL-17, and IL-18 in patients with active psoriasis and correlation with disease severity. Mediators Inflamm 2005; 200: 273-9.

40. Perry D. The current concept of Th17 cells and their expanding role in systemic lupus erythematous. Arthritis 2011; 49: 1-10.

41. Zhao L, Jiang Z, Jiang Y, et al. IL-22+CD4+ T-cells in patients with active systemic lupus erythematosus. Exp Biol Med (Maywood) 2013; 238: 193-9.
42. Pernis B. Th17 cells in rheumatoid arthritis and systemic lupus erythematous. J Internal Med 2009; 265: 644-52.

43. Li L, Boussiotis VA. The role of IL-17-producing Foxp3 (+) CD4 (+) T cells in inflammatory bowel disease and colon cancer. Clin Immunol 2013; 148: 246-53.

44. Dige A, Støy S, Rasmussen TK, et al. Increased levels of circulating Th17 cells in quiescent versus active Crohn's disease. J Crohns Colitis 2013; 7: 248-55.

45. Siakavellas SI, Bamias G. Role of the IL-23/IL-17 axis in Crohn's disease. Discov Med 2012; 14: 253-62.

46. Brembilla NC, Chizzolini C. T cell abnormalities in systemic sclerosis with a focus on Th17 cells. Eur Cytokine Netw 2012; 23: 128-39.

47. Zepp J, Wu L, Li X. IL-17 receptor signaling and T helper 17-mediated autoimmune demyelinating disease. Trends Immunol 2011; 32: 232-9.

48. Aranami T, Yamamura T. Th17 cells and autoimmune encephalomyelitis (EAE/MS). Allergol Inter 2008; 57: 115-20.

49. Takehara K, Sato S. Localized scleroderma is an auto immune disorder. Rheumatology 2005; 44: 274-9.

50. Onishi RM, Gaffen SL. Interleukin-17 and its target genes: mechanisms of interleukin-17 function in disease. Immunology 2010; 129: 311-21.

51. Dańczak-Pazdrowska A, Kowalczyk M, Szramka-Pawlak B, et al. Interleukin-17A and interleukin-23 in morphea. Arch Med Sci 2012; 8: 1089-95.

52. Kurasawa K, Hirose K, Sano H, et al. Increased interleukin 17 production in patients with systemic sclerosis. Arthritis Rheum 2000; 43: 2455-63.

53. Deleuran B, Abraham D. Possible implication of the effector CD4+ T-cell subpopulation TH17 in the pathogenesis of systemic scleroderma. Nat Clin Pract Rheumatol 2007; 3: 682-3.

54. Nakashima T, Masatoshi J, Keitaro Y, et al. Impaired IL-17 signaling pathway contributes to the increased collagen expression in scleroderma fibroblasts. J Immunol 2012; 188: 3573-83.

55. von Vietinghoff S, Ley K. Interleukin 17 in vascular inflammation. Cytokine Growth Factor Rev 2010; 21: 463-9.

56. Shahneh FZ, Babaloo Z, Baradaran B, et al. Behçet's syndrome in Iranian Azari people. Pak J Biol Sci 2012; 15: 1045-7.

57. Shahneh FZ, Babaloo Z, Baradaran B, Sepehr KS. Insights into Behçet's disease. Postep Derm Alergol 2012; 29: 461-6.

58. Shahneh FZ, Babaloo Z, Baradaran B, et al. New insights of HLA class I association to Behçet's syndrome in Iranian Azari patients. Autoimmunity Highlight 2013; 4: 101-2.

59. Geri G, Terrier B, Rosenzwajg M. Critical role of IL-21 in modulating Th17 and regulatory T cells in Behcet disease. J Aller Clin Immunol 2011; 128: 655-64.

60. Schuijs MJ, Willart MA, Hammad H, Lambrecht BN. Cytokine targets in airway inflammation. Curr Opin Pharmacol 2013; 13: 351-61.

61. Ma CS, Chew GY, Simpson N. Deficiency of Th17 cells in hyper IgE syndrome due to mutation in STAT3. J Exp Med 2008; 205: 1551-7. 